\title{
Cochlear Compression: Perceptual Measures and Implications for Normal and Impaired Hearing
}

\author{
Andrew J. Oxenham and Sid P. Bacon
}

This article provides a review of recent developments in our understanding of how cochlear nonlinearity affects sound perception and how a loss of the nonlinearity associated with cochlear hearing impairment changes the way sounds are perceived. The response of the healthy mammalian basilar membrane (BM) to sound is sharply tuned, highly nonlinear, and compressive. Damage to the outer hair cells (OHCs) results in changes to all three attributes: in the case of total OHC loss, the response of the BM becomes broadly tuned and linear. Many of the differences in auditory perception and performance between normal-hearing and hearingimpaired listeners can be explained in terms of these changes in BM response. Effects that can be accounted for in this way include poorer audiometric thresholds, loudness recruitment, reduced frequency selectivity, and changes in apparent temporal processing. All these effects can influence the ability of hearing-impaired listeners to perceive speech, especially in complex acoustic backgrounds. A number of behavioral methods have been proposed to estimate cochlear nonlinearity in individual listeners. By separating the effects of cochlear nonlinearity from other aspects of hearing impairment, such methods may contribute towards identifying the different physiological mechanisms responsible for hearing loss in individual patients. This in turn may lead to more accurate diagnoses and more effective hearing-aid fitting for individual patients. A remaining challenge is to devise a behavioral measure that is sufficiently accurate and efficient to be used in a clinical setting.

(Ear \& Hearing 2003;24;352-366)

One of the most impressive features of the auditory system is its wide dynamic range. In the midfrequency region, between approximately 1 and 3 $\mathrm{kHz}$, listeners with normal hearing can detect pressure variations of approximately $0.00002 \mathrm{~Pa}$, and yet pressures one million times greater than this can be reached before the sensation becomes universally

Research Laboratory of Electronics (A.J.O.), Massachusetts Institute of Technology, Cambridge, Massachusetts; Speech and Hearing Bioscience and Technology Program (A.J.O.), Harvard-MIT Division of Health Sciences and Technology, Cambridge, Massachusetts; and Psychoacoustics Laboratory, Department of Speech and Hearing Science (S.P.B.), Arizona State University, Tempe, Arizona.

DOI: 10.1097/01.AUD.0000090470.73934.78 painful or damaging. Imagine a mechanical sensing system with that kind of sensitivity and range: the same system that could detect movements as small as $1 \mathrm{~mm}$ (less than 0.05 inches) would also be able to cope with movements as large as $1 \mathrm{~km}$, or more than half a mile. The visual system also has a large dynamic range but, as anyone going from a bright to a dark room will testify, vision can take several minutes to return to maximum sensitivity after strong stimulation. In contrast, the auditory system recovers its full sensitivity within a fraction of a second from all but the most intense sounds.

The auditory system's frequency selectivity, or ability to resolve or separate out sounds of different frequencies, also sets it apart from the visual system. In vision, we cannot see two colors in the same place at once: if two (or three in the case of television) light sources emanate from the same location, they are fused into a single perceptual source with a color that is derived from a combination of the individual frequencies. In contrast, the auditory system is able to hear many different frequencies at once, from the harmonics within the voice of a single speaker, which give the voice its pitch and timbre, to the myriad of sound when a full orchestra plays. Put another way, in contrast to the visual system, there is no combination of one, two, or three sinusoids that could be presented to fool a listener into thinking a whole orchestra was playing.

The auditory system's large dynamic range and exquisite frequency selectivity, both of which form vital components of our ability to communicate in the acoustic environment, are largely established by the mechanoelectrical transduction that takes place in the cochlea. In particular, the healthy cochlea responds to sound in a way that is highly nonlinear and compressive. The extent of these nonlinearities and their origins in the mechanics of the basilar membrane (BM) has only become fully established in approximately the past decade. Of particular interest are the questions of how cochlear nonlinearities affect perception, and whether many of the challenges faced by people with hearing impairments can be accounted for in terms of changes in cochlear mechanics. This review does not attempt to provide a comprehensive review of all the literature pertaining to the perceptual consequences of cochlear nonlinearity. Instead, the basic principles are

0196/0202/03/2405-0352/0 • Ear \& Hearing • Copyright @ 2003 by Lippincott Williams \& Wilkins • Printed in the U.S.A. 
set out and are illustrated by some of the most pertinent recent studies, with an emphasis on the points most relevant for hearing impairment. The interested reader is referred elsewhere for fuller literature surveys (Bacon \& Oxenham, in press; Moore \& Oxenham, 1998; Oxenham \& Bacon, in press).

\section{Nonlinear Cochlear Transduction at the Characteristic Frequency}

When a sound wave reaches the ear drum, it is transmitted via the vibrations of the middle-ear bones to the oval window of the cochlea. There it sets up a pattern of vibration along the cochlear partition, including the BM. The response to a pure tone is a traveling wave along the BM. It is primarily the frequency of the tone that determines where along the BM the traveling wave reaches its peak, although the tone's intensity (or level) also has some effect. At low sound levels, the frequency that produces the greatest response at a given point along the $\mathrm{BM}$ is known as the characteristic frequency (CF) of that point.

One of the most important findings for hearing research in recent times has been the discovery of the "cochlear amplifier." This phrase refers to the properties of the cochlea that are physiologically vulnerable and lead to high sensitivity and sharp tuning. It is now established in animal studies that these properties are already found at the level of the BM (Narayan, Temchin, Recio, \& Ruggero, 1998; Rhode, 1971; Sellick, Patuzzi, \& Johnstone, 1982). Although the precise mechanisms are still somewhat contentious, it is generally accepted that the $\mathrm{OHCs}$, which form three (or more) rows along the length of the BM, are responsible for the highly nonlinear gain of the cochlear amplifier (Ruggero \& Rich, 1991; for a recent review, see Robles \& Ruggero, 2001).

Figure 1 shows the response of one point along a chinchilla's BM in response to a tone at the CF of that point, taken from data of Ruggero, Rich, Recio, Narayan, and Robles (1997). The solid line shows the response when the cochlea is healthy; the dashed line shows the response after death, when the OHCs are no longer functioning normally [similar, although typically less severe, effects are observed when the OHCs are adversely affected by, for example, acoustic overstimulation or the intravenous injection of the ototoxic drug quinine (Ruggero, Rich, Robles, \& Recio, 1996)]. Two points are worthy of mention here. First, the healthy cochlea is much more sensitive to low-level sounds; the BM starts to produce measurable vibrations at much lower sound input levels than is the case for the damaged cochlea. Second, the response of the healthy cochlea is

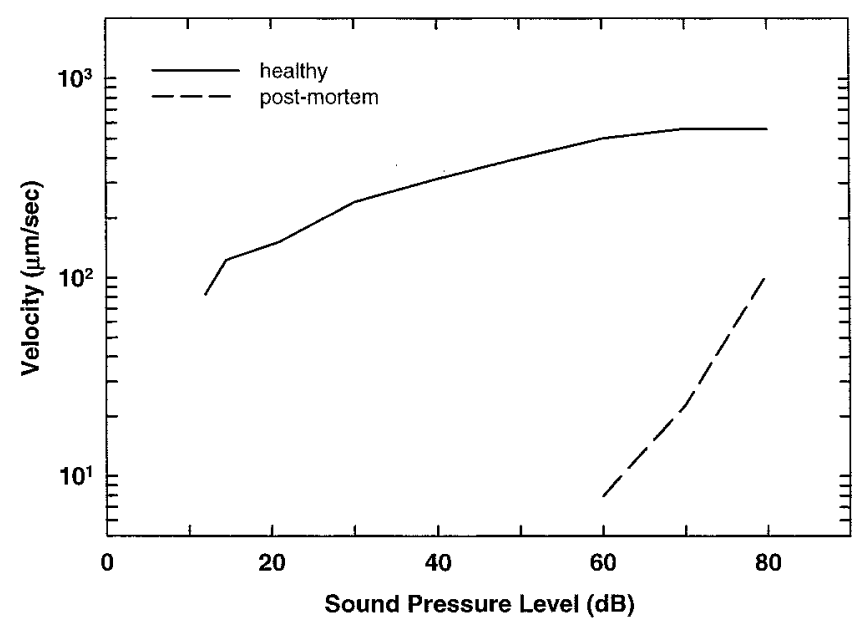

Figure 1. Basilar membrane input-output functions from the basal region of a chinchilla cochlea in response to a tone at the CF of the place of measurement. The data were obtained when the cochlea was healthy (solid line) and after death, when the OHCs and hence the cochlea were no longer functioning normally (dashed line). The data are taken from Ruggero et al. (1997), with permission from the author and the American Institute of Physics.

highly compressive; in the mid-level region a change in input sound pressure of $50 \mathrm{~dB}$ (from 30 to $80 \mathrm{~dB}$ SPL) leads to a change of slightly less than $10 \mathrm{~dB}$ in the velocity of the BM. (A change in velocity by a factor of 10 corresponds to a $20-\mathrm{dB}$ change in response.) This is equivalent to a compression ratio of approximately $5: 1$, compared to the essentially linear (1:1) relationship between sound pressure and $\mathrm{BM}$ velocity in the case of the damaged cochlea (dashed line). The compression implies that the gain of the cochlear amplifier at low levels is much greater than its gain at high levels. At low, and possibly very high, levels the response of the healthy cochlea is more linear (Ruggero, Rich, \& Recio, 1996).

Using just the information presented in Figure 1, we can already make some predictions about how a loss of cochlear nonlinearity might affect perception. Figure 2 provides a schematic representation of the data in Figure 1. Let us assume for simplicity that the response of the $\mathrm{BM}$ at that point determines how we perceive tones at the $\mathrm{CF}$. The two horizontal dotted lines represent two levels of BM vibration. The lower one, marked $\mathrm{T}$ for threshold, represents the minimum level of vibration necessary for sound to be detected; the upper one, marked U for uncomfortable, represents the maximum level of vibration permitted before the sound becomes too loud for comfort. One can think of the space between the points as the full range of loudness, going from just audible (T) to too loud (U). The point along the abscissa at which each horizontal line intersects 


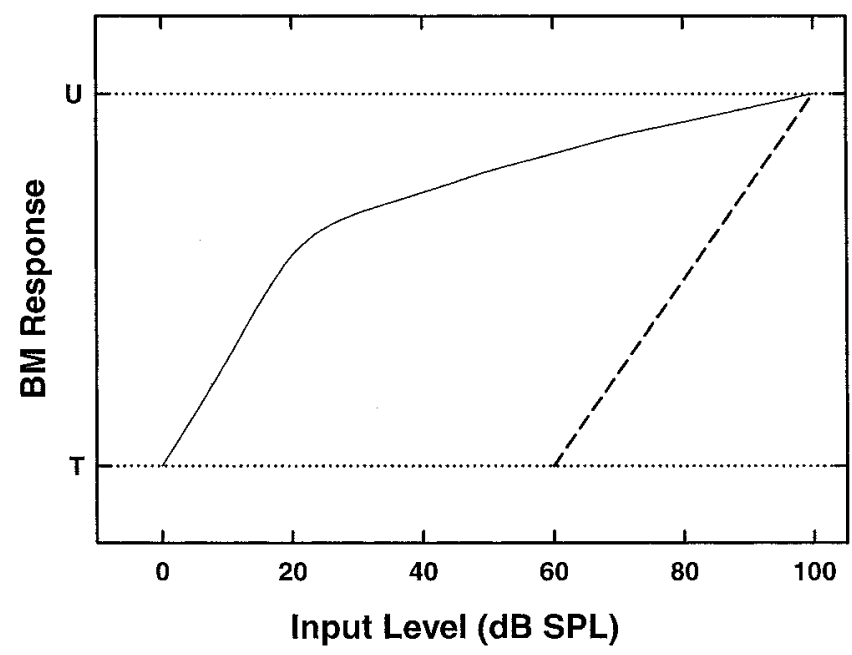

Figure 2. Schematic diagram of a BM input-output function for a normal (solid line) and abnormal (dashed line) cochlea. The difference between the two functions represents the amount of gain provided by the OHCs. Along the ordinate, T represents threshold and $U$ represents uncomfortable loudness.

with the response curve gives the relevant sound input level. For the linear BM response (damaged cochlea; dashed line), the "threshold" line is reached at a level of $60 \mathrm{~dB}$ SPL. As the uncomfortable level of $\mathrm{BM}$ vibration is reached at a sound level of $100 \mathrm{~dB}$ SPL, the dynamic range of this system is only $40 \mathrm{~dB}$. The nonlinear gain associated with the compressive BM response (healthy cochlea; solid curve) has a dramatic effect on the dynamic range. The uncomfortable level is still reached at approximately the same level of $100 \mathrm{~dB}$ SPL, but the threshold line is crossed at the much lower level of $0 \mathrm{~dB}$ SPL, giving a total dynamic range of $100 \mathrm{~dB}$. This is achieved by the large gain or amplification at low (but not high) levels and the resultant compression.

If the two lines represented the BM input-output functions of two listeners, one normal-hearing (solid line) and one hearing-impaired (dashed line), the hearing-impaired listener would have a hearing loss of approximately $60 \mathrm{~dB}$ at that frequency, but would still find sounds of $100 \mathrm{~dB}$ SPL or more to be uncomfortable. This simple illustration immediately provides a cochlea-based explanation for some of the most common symptoms of cochlear hearing loss, namely elevated absolute thresholds ( $\mathrm{T}$ reached at a higher sound input level than in normal hearing), and loudness recruitment ( $\mathrm{U}$ reached at a similar level to normal hearing, despite an elevated T). Loudness recruitment refers to the observation that loudness level often grows more rapidly with increasing stimulus level in hearing-impaired listeners than it does in normal-hearing listeners. An analog of this can be seen in Figure 2 in that the input-output function of the damaged cochlea is generally steeper than that of the healthy cochlea. Interestingly, one prediction of this explanation of loudness recruitment is that the growth of loudness level near threshold should be similar for both normal and impaired hearing. This is because both have roughly linear input-output functions near threshold. This prediction is in line with recent findings of Buus and Florentine (2001), who found that slopes for loudness level growth near threshold were similar for normal-hearing and hearing-impaired listeners. The possible influence of the BM in determining loudness recruitment was suggested by Yates, Winter, and Robertson (1990), and similar explanations based on steeper auditory-nerve rateintensity functions in damaged cochleae (Evans, 1974) were suggested by Harrison (1981), before it was clear that these effects were mediated directly by $\mathrm{BM}$ mechanics.

If nonlinear amplification and compression (Fig. 2) were the extent of the cochlea's processing of sound, it would be a relatively simple matter to compensate for hearing loss. A compression hearing aid could be devised with an input-output characteristic similar to that shown by the solid line in Figure 2. Because cochlear compression is believed to be fast acting (Ruggero, Robles, Rich, \& Recio, 1992), the time constant of the compression hearing aid would need to be very short. However, although compression hearing aids are generally believed to provide better comfort for hearing-impaired listeners, there is little evidence so far that they generally provide gains in speech intelligibility, particularly in noisy environments (Levitt, in press), and no one has claimed that such aids restore hearing to normal. Of the many possible reasons why a simple compression hearing aid does not fully compensate for hearing loss, one important reason may be that the compression observed in the cochlea is frequency selective, at least at medium to high frequencies. The following section addresses this issue.

\section{Frequency and Level Effects in the Cochlea}

Figure 3 shows the response of one point along the chinchilla BM to tones of various frequencies (Ruggero et al., 1997). The different curves represent tones of different levels, ranging from $10 \mathrm{~dB}$ to $90 \mathrm{~dB}$ SPL. The $\mathrm{CF}$ of the measurement point is defined as the frequency that produces the greatest response at low levels, in this case $10 \mathrm{kHz}$. Two features of the graph are especially important. First, the tuning is sharpest at low levels; the response region widens considerably at high levels. Second (and related), the response growth (i.e., the change in response as a function of input level) varies with 


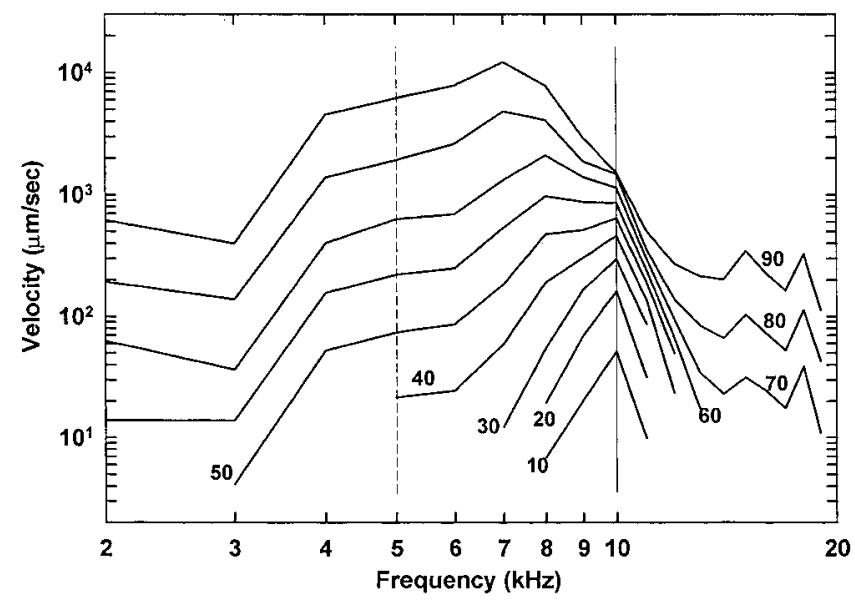

Figure 3. The response of the chinchilla $B M$ at a $C F$ of $10 \mathrm{kHz}$ in response to a fixed-level tone with a frequency represented along the abscissa. The level of the tone varied from 10 to 90 dB SPL. Vertical lines mark the responses to a tone at either 5 or $10 \mathrm{kHz}$ (see Figure 4). The data are taken from Ruggero et al. (1997), with permission from the author and the American Institute of Physics.

frequency: for frequencies well below $\mathrm{CF}$, the response growth is roughly linear, so that a $10-\mathrm{dB}$ change in sound pressure level results in a $10-\mathrm{dB}$ change in $\mathrm{BM}$ response; for frequencies around $\mathrm{CF}$ the growth is highly compressive. This second feature is illustrated by re-plotting some of the results in Figure 3. In particular, the results at 5 and 10 $\mathrm{kHz}$ (see vertical lines in Fig. 3) are plotted in Figure 4 as input-output functions. That is, the response of the BM is plotted as a function of input sound level

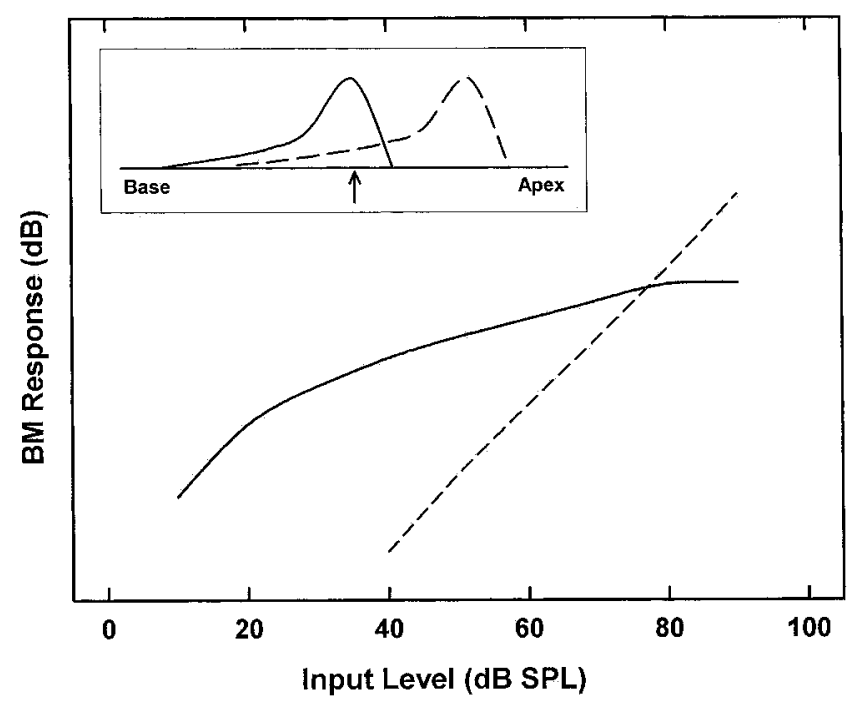

Figure 4. Basilar membrane input-output function for a tone at CF (10 kHz; solid line) and a tone one octave below CF (5 $\mathrm{kHz}$; dashed line), taken from the data in Figure 3. The inset is a cartoon of the traveling wave envelopes for the two tones. The arrow indicates the measurement place. for a tone with a frequency one octave below the CF (dashed line) and for a tone at the CF (solid line). The inset in Figure 4 provides a schematic diagram of the cochlea's response to the two tones at one input level. The envelopes of the traveling waves for the tone at $\mathrm{CF}$ and the tone one octave below $\mathrm{CF}$ are shown as solid and dashed lines, respectively. The arrow shows the point at which the measurements are occurring. The input-output functions in Figure 4 show how the response to a tone at CF is highly compressive (same as in Fig. 1), but also show that the response to a tone well below $\mathrm{CF}$ is linear. In other words, the amount of compression applied to the incoming sound at each place along the BM depends on the relationship between the $\mathrm{CF}$ and the frequency content of the sound.

The effect of cochlear damage, in particular damage to the OHCs, is to reduce or eliminate the level dependence of frequency tuning at the BM, such that the overall response becomes linear. Now, at each level the tuning remains the same and the overall response to sound grows linearly as a function of sound level. The tuning found in the damaged cochlea often resembles the broad tuning found at the highest sound levels in the normal cochlea (see the upper-most curve in Fig. 3). Reduced frequency selectivity is a common finding in listeners with cochlear hearing impairment: such listeners typically exhibit broader tuning in tasks measuring the shape of the auditory filters (Dubno \& Schaefer, 1995; Florentine, Buus, Scharf, \& Zwicker, 1980; Leek \& Summers, 1993; Moore, 1985; Tyler, Hall, Glasberg, Moore, \& Patterson, 1984). Thus, it is likely that the poorer frequency selectivity found in such listeners is caused by broader cochlear tuning.

In summary, this and the previous section have shown that elevated audiometric thresholds, loudness recruitment, and poor frequency selectivity are likely to be direct consequences of a loss of cochlear nonlinearity. The reasoning behind this is fairly intuitive: reduced cochlear gain leads to elevated thresholds; steeper input-output functions lead to steeper growth of loudness level; and broader cochlear tuning leads to poorer frequency selectivity. In contrast, the link between cochlear hearing loss and temporal processing is less immediately clear. This topic is addressed in the following section.

\section{Temporal Processing: Influence of Cochlear Nonlinearity}

Sounds are by their nature dynamic, changing over time in terms of level and spectral content. The process of following rapid changes, and integrating information, over time is referred to as temporal processing. Many different psychoacoustic measures 
of temporal processing have been devised over the years. For instance, gap-detection tasks test listeners' abilities to follow rapid changes over time by measuring the shortest interval of silence that is detectable in an otherwise continuous sound; modulation-detection tasks measure how listeners' abilities to perceive rapid fluctuations (or modulation) change as the rate of modulation is varied; and forward-masking tasks can measure how rapidly the thresholds for a brief signal recover after stimulation by a masking sound. All these measures are concerned with the limits of our ability to follow rapid changes and are collectively referred to as measures of temporal resolution. Other tests measure the longest time span over which information can be efficiently combined by, for instance, measuring how the detection threshold for a tone improves as the duration of the tone increases. Such tests are thought to measure the limits of temporal integration. Historically, the two classes of temporal processing, short-term temporal resolution and longterm temporal integration, have been treated separately and it has proved difficult to unite them within one theoretical framework (de Boer, 1985), although recent models can deal with both classes of phenomena (Dau, Püschel, \& Kohlrausch, 1996; Viemeister \& Wakefield, 1991).

There are numerous studies on the effects of hearing impairment on temporal processing (Bacon \& Gleitman, 1992; Bacon \& Viemeister, 1985; Fitzgibbons \& Gordon-Salant, 1987; Irwin, Hinchcliff, \& Kemp, 1981; Snell \& Frisina, 2000), as well as some that attempt to understand the results within a theoretical framework of known physiological changes (Derleth, Dau, \& Kollmeier, 2001; Glasberg \& Moore, 1992; Oxenham \& Moore, 1997; Oxenham, Moore, \& Vickers, 1997). Overall, the pattern of results seems somewhat confusing at first glance. Certain measures of temporal processing, such as forward masking, gap detection in narrowband noise, and temporal integration, show strong effects of hearing impairment, whereas others, such as modulation detection and gap detection in tones, often remain unaffected. One effect of cochlear hearing loss-loss of frequency selectivity-has in the past been proposed as a possible theoretical reason for differences in performance between normalhearing and hearing-impaired listeners. This is because within a simple resonant filter system, frequency and temporal resolution are restricted by the same theoretical limit; within this limit, better frequency resolution implies poorer temporal resolution, and vice versa. The theoretical prediction is, therefore, that hearing-impaired listeners with poorer frequency resolution should actually have better temporal resolution than normal-hearing lis- teners. Although there are cases at very low sensation levels of hearing-impaired listeners performing somewhat better than normal-hearing listeners, this has generally not been found to be the case; if anything, hearing impairment can lead to a deterioration in certain measures of temporal resolution (Moore, 1998). The reason for this is probably that temporal resolution is limited by processing stages higher than the cochlea for all but the lowest frequencies. Thus, changes in frequency resolution do not lead to the changes in temporal resolution predicted by a simple single-filter model.

Another effect of hearing impairment, which is probably of more direct relevance to temporal processing, is the loss of cochlear compression. In fact, it can be shown that many of the changes in temporal processing can be accounted for by known changes in cochlear compression. Two examples, gap detection in narrowband noise and forward masking, are described below. For other examples of the effects of cochlear compression on temporal resolution and integration, see Oxenham et al. (1997) and Derleth et al. (2001).

Thresholds for the detection of temporal gaps in sinusoids are similar for normal-hearing and hearing-impaired listeners when compared at equal sensation levels (Moore \& Glasberg, 1988). However, gaps in narrowband noise are often much more poorly detected by hearing-impaired listeners (Fitzgibbons \& Wightman, 1982; Glasberg, Moore, \& Bacon, 1987). Glasberg et al. (1987) and Moore and Glasberg (1988) suggested a link between poor gapdetection performance in narrowband noise and loudness recruitment. Cast in terms of cochlear mechanics, their suggestion amounts to the idea that a loss of compression would lead to the inherent level fluctuations in the narrowband noise being exaggerated and consequently more easily confused with the gap itself. Figure 5 illustrates the idea. The two panels both show the temporal envelope of the same narrowband noise. However, in the upper panel the envelope has been compressed (4:1 compression), whereas the lower panel shows the unaltered envelope, thereby simulating the effects of normal cochlear compression (upper panel) and of an impaired, linear cochlea (lower panel). Although both panels of Figure 5 show the inherent fluctuations in the noise, the effect is clearly more noticeable in the lower panel. What is more, there are several epochs in the lower panel where the level drops more than $10 \mathrm{~dB}$ below the peak for several milliseconds at a time. It is easy to see why such inherent fluctuations might be confused with an actual gap in the noise.

Glasberg and Moore (1992) confirmed the hypothesized effects of compression and lack thereof. They 

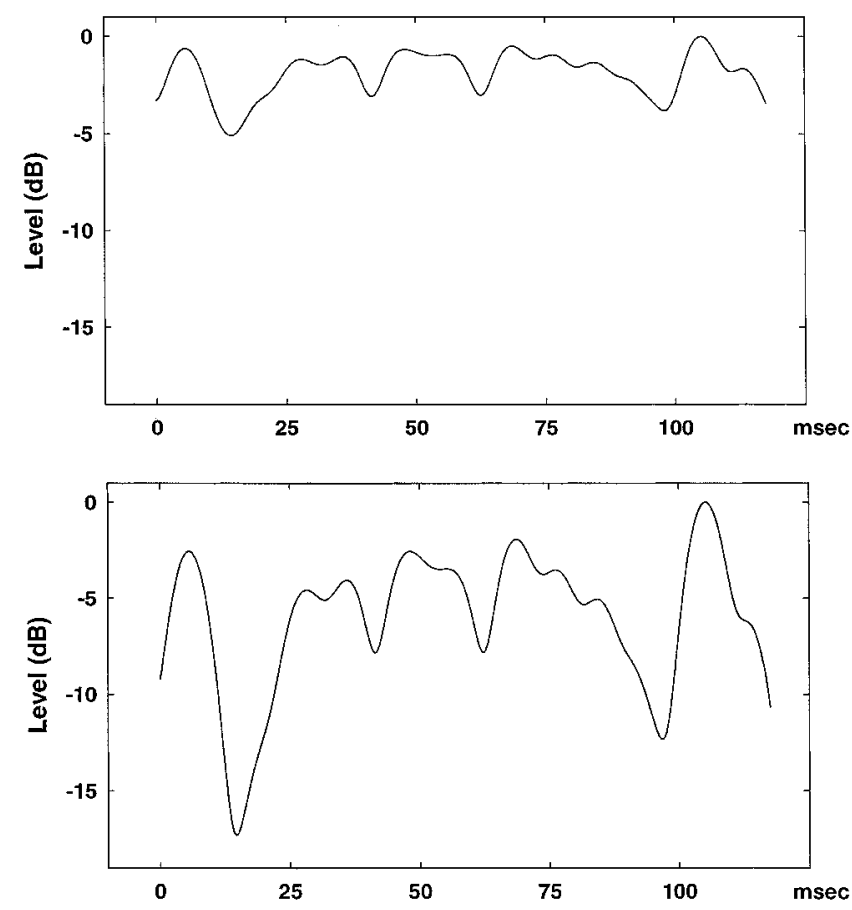

Figure 5. The temporal envelope of a narrowband noise. The upper panel shows the envelope after 4:1 compression and the lower panel shows the same envelope without compression (1:1). The envelope processing represents normal (upper panel) and impaired (lower panel) hearing.

manipulated narrowband-noise carriers by raising the envelopes to the power of 0.5 (compression), 1 (linear, no change), 1.5, and 2 (expansion). They found that increasing the exponent led to poorer gap detection. Also, an exponent of 2 was often sufficient to produce thresholds in normal-hearing listeners that were similar to those of the hearing-impaired listeners in the linear condition. Similarly, thresholds in the hearing-impaired listeners for the 0.5 exponent approached those of the normal-hearing listeners in the linear condition. These results are consistent with the idea that cochlear nonlinearity affects gap detection with fluctuating stimuli and that a loss of compression leads to poorer performance.

Forward masking is the elevation in signal thresholds produced by a masker after its termination. A curve tracing how forward-masking thresholds decrease after the end of a masker shows the speed with which the auditory system recovers from stimulation, and so forward masking can be thought of as a measure of temporal resolution. Figure 6 shows a schematic model of how forward masking might occur. The first two stages (filtering and nonlinearity) are a simplifying approximation of the nonlinear filtering and transduction of the cochlea, up to and including the inner hair cells (IHCs). The third stage is a sliding temporal integrator, or "temporal window." This stage has the function of

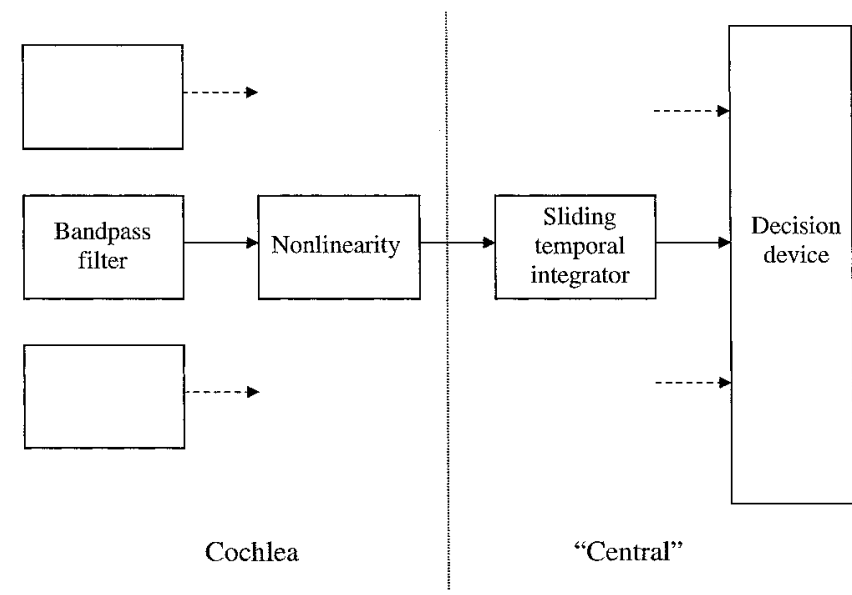

Figure 6. A schematic model of temporal processing, consisting of the following four stages: (1) bandpass filtering; (2) compression and rectification; (3) temporal smoothing; and (4) a decision device.

smoothing the incoming stimulus, so that rapid fluctuations are attenuated but slow fluctuations are passed almost unchanged. The temporal window has no known physiological basis, but it is posited to be at a high (cortical?) level of the auditory pathways, where neurons with long time constants are not uncommon (Eggermont, 2002). The final stage is the decision device, where information from the output of the temporal window is assessed to perform the task at hand, such as deciding whether or not a signal was present with the masker. The duration and shape of the temporal window influence the rate at which forward masking is predicted to recover.

Hearing-impaired listeners generally show a more gradual recovery from forward masking than do normal-hearing listeners, when forward maskers with the same sound pressure level are used for both groups. Although the difference is reduced when the maskers are equated in terms of sensation level (Glasberg et al., 1987), the more gradual recovery from forward masking has been quantified in terms of longer time constants for hearing-impaired listeners, implying impaired temporal resolution (Holube \& Kollmeier, 1996; Nelson \& Freyman, 1987). In fact, it is likely that many of the differences between normal and impaired hearing in forward masking can be accounted for by changes in cochlear nonlinearity (Fig. 6; model stage 2) rather than changes in the time constants of the temporal window (model stage 3). This is illustrated in Figure 7, the left panel of which shows the output of the temporal window in response to a forward masker. Between times $t_{1}$ and $t_{2}$ the output of the temporal window has decayed by $2 \mathrm{~dB}$. By assuming that the signal threshold and the masker level are proportional at the output of the 
Internal effect of masker

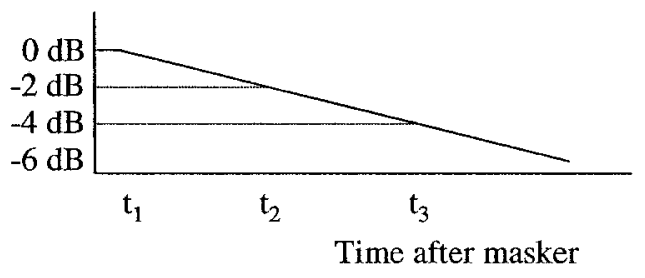

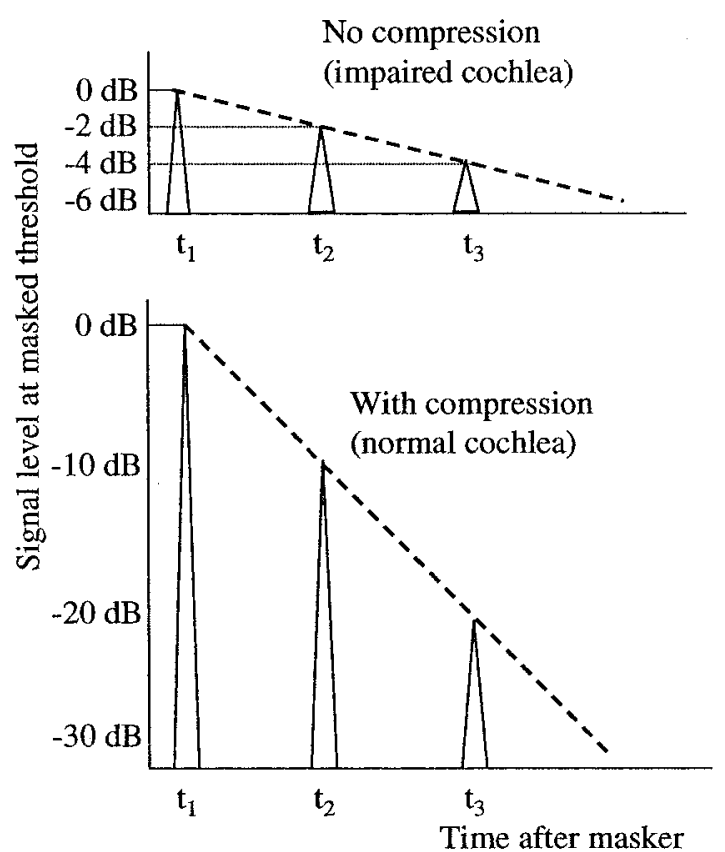

Figure 7. Forward masking. The left panel shows how the internal representation of the masker decays after the masker has been turned off. Between times $t_{1}$ and $t_{2}$ the masker representation has decayed by $2 \mathrm{~dB}$. The upper and lower right panels show the changes in signal levels needed to match the decay of masker excitation for an impaired and healthy cochlea, respectively. In the impaired case (upper panel), the signal is processed linearly and so a 2-dB change in masker excitation is matched by a 2-dB change in signal level. In the normal case (lower panel), the signal is compressed and so a 10-dB change in signal level is required to match the 2-dB change in masker excitation.

temporal window, we can predict the signal level at threshold. In the case of the impaired cochlea, we can assume that the BM processes sound linearly, and so a $2-\mathrm{dB}$ decrease in masker output can be matched by a $2-\mathrm{dB}$ decrease in signal level. In other words, for the impaired ear the signal threshold decreases by $2 \mathrm{~dB}$ as the signal is moved from $t_{1}$ to $t_{2}$, as shown in the upper right panel of Figure 7. In the case of the healthy cochlea, the response of the BM is compressive. If 5:1 compression is assumed, the signal level has to be decreased by $10 \mathrm{~dB}$ to produce a $2-\mathrm{dB}$ change in the response to the signal. Thus, for the normal ear the signal threshold decreases by $10 \mathrm{~dB}$ as the signal is moved from $t_{1}$ to $t_{2}$. This is a much greater change than is predicted for the impaired ear. Note, however, that what appears to be a more rapid recovery from forward masking has nothing to do with the temporal window itself; rather it is the compressive nonlinearity that changes the effective temporal characteristics of forward masking.

Oxenham and Moore (1997) tested the idea that changes in forward masking with hearing impairment were caused by changes in cochlear compression. In a group of normal-hearing listeners, they measured the masker levels necessary to just mask a signal for a variety of signal levels and maskersignal gaps. The data from the experiment are shown as symbols in the left panel of Figure 8. The data were used to derive temporal window parameters for the model shown in Figure 6. The nonlinearity in the model was designed to simulate the compression found in a healthy cochlea (Fig. 1). The predictions of the model are shown as solid lines in the left panel of Figure 8. The same conditions were tested in three hearing-impaired listeners, all with moderate-to-severe hearing loss of cochlear origin. Their mean data are shown as symbols in the right panel of Figure 8, and the model predictions are shown as solid lines. It can be seen that the predictions lie reasonably close to the data. The important point, however, is that the model parameters were not fitted to the hearing-impaired data. Instead, only the compressive nonlinearity was removed. Thus, with the same temporal window, the model can predict the changes in forward masking caused by hearing impairment simply by assuming a loss of cochlear nonlinearity. This conclusion has since been confirmed by Derleth et al. (2001).

In summary, a number of aspects of temporal processing in hearing-impaired listeners can be explained in terms of a loss of cochlear nonlinearity, in particular the loss of compression. Thus, level effects (loudness recruitment, loss of audibility), frequency effects (poorer frequency selectivity), and temporal effects (effectively poorer temporal resolution) in 

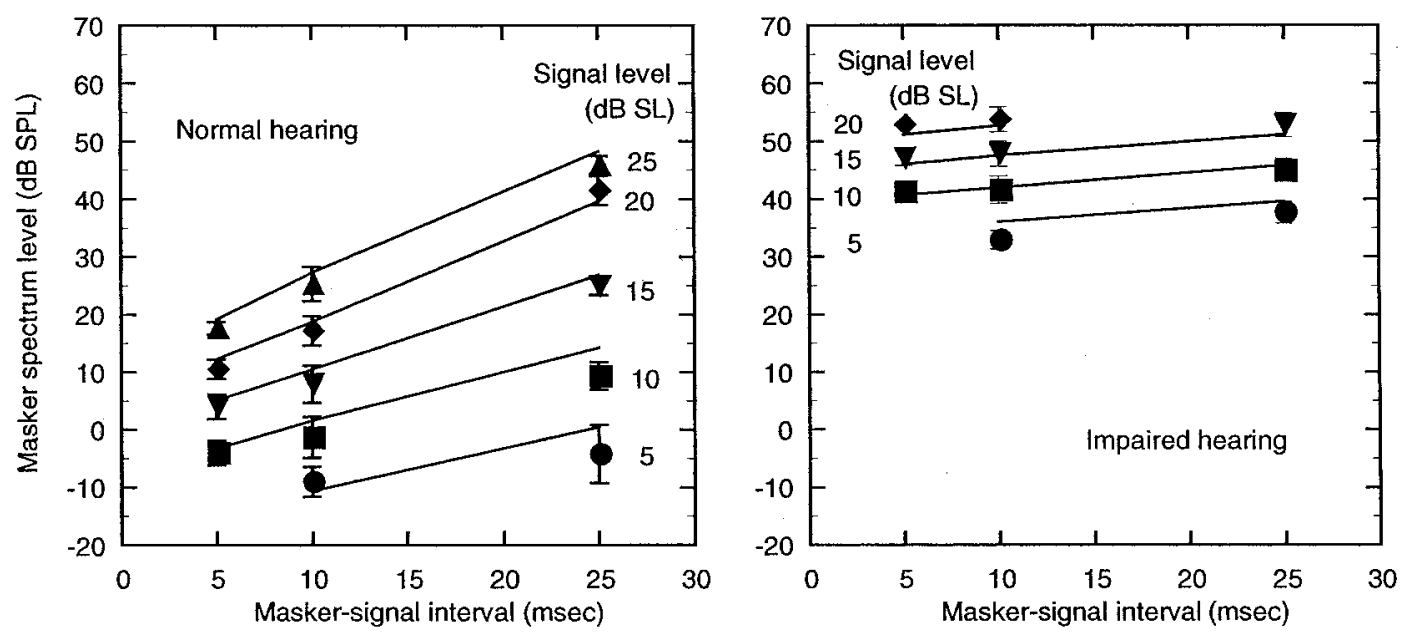

Figure 8. Forward-masking data (symbols) and predictions from the temporal-window model (lines), taken from Oxenham and Moore (1997). The left panel shows mean data from normal-hearing listeners and the right panel shows mean data from three listeners with moderate-to-severe cochlear hearing loss in the same conditions. The model predictions for the hearing-impaired listeners were derived by simply removing the nonlinear stage from the model (see Fig. 6) used with the normal-hearing listeners' data.

hearing impairment can all be understood in terms of changes in cochlear mechanics, due presumably to OHC dysfunction, damage, or loss.

\section{Real-World Correlates of Psychoacoustic Results}

From a theoretical standpoint, it is satisfying to be able to tie together the results from a variety of psychoacoustic tasks and explain them all using the same underlying mechanism. But from a more practical viewpoint, what do these psychoacoustic tasks tell us about how patients cope in their everyday acoustic environments? Although the relationship between psychoacoustic performance and real-world listening performance in, for example, speech reception is not fully understood, there are a number of clear parallels, some more obvious than others.

The obvious correlate to elevated audiometric thresholds is an inability to hear low-level sounds, which can lead to difficulties in quiet conversational situations. Loudness recruitment, or the abnormal relationship between loudness and intensity, also has clear everyday implications. For instance, recruitment is one reason why simple linear amplification in hearing aids is not sufficient; the amplification necessary to make quiet sounds audible will result in loud sounds being uncomfortably loud.

The effects of poorer frequency selectivity are less obvious and are not fully understood. However, it is becoming clear through simulation studies that poorer frequency selectivity, although perhaps not greatly affecting speech reception in quiet, can have profound effects on the ability to understand speech in a noisy background (Baer \& Moore, 1993; ter
Keurs, Festen, \& Plomp, 1992, 1993). A recent study, which manipulated frequency resolution in a simulation of cochlear-implant perception, found that the effects of poorer frequency resolution were especially apparent when speech was presented in a complex acoustic background, such as a competing talker or an amplitude-modulated noise (Qin \& Oxenham, 2003). Thus, good frequency selectivity may be crucial in complex acoustic situations, such as the well-known "cocktail party" (Cherry, 1953), where listeners are required to segregate different sources that are present at one time.

The consequences of poor temporal resolution may include a reduced ability to take advantage of momentary dips in the level of background sounds to hear out the sounds of interest. This may be particularly important in real-world situations, where background noises are often not continuous, steadystate sounds, but fluctuate from moment to moment.

One illustration of how hearing impairment can affect speech reception was provided by Peters, Moore, and Baer (1998). They measured speech reception thresholds with normal-hearing and hearing-impaired listeners in a variety of noise backgrounds, including steady-state noise, temporally modulated noise, noise with spectral dips, modulated noise with spectral dips, and a single-talker interferer (with temporal modulations and timevarying spectral peaks and dips). For the normalhearing listeners, the authors confirmed that listeners benefited greatly from both spectral and temporal dips in the masking noise; young listeners' thresholds improved by more than $6 \mathrm{~dB}$ when the noise was temporally modulated and by more than 

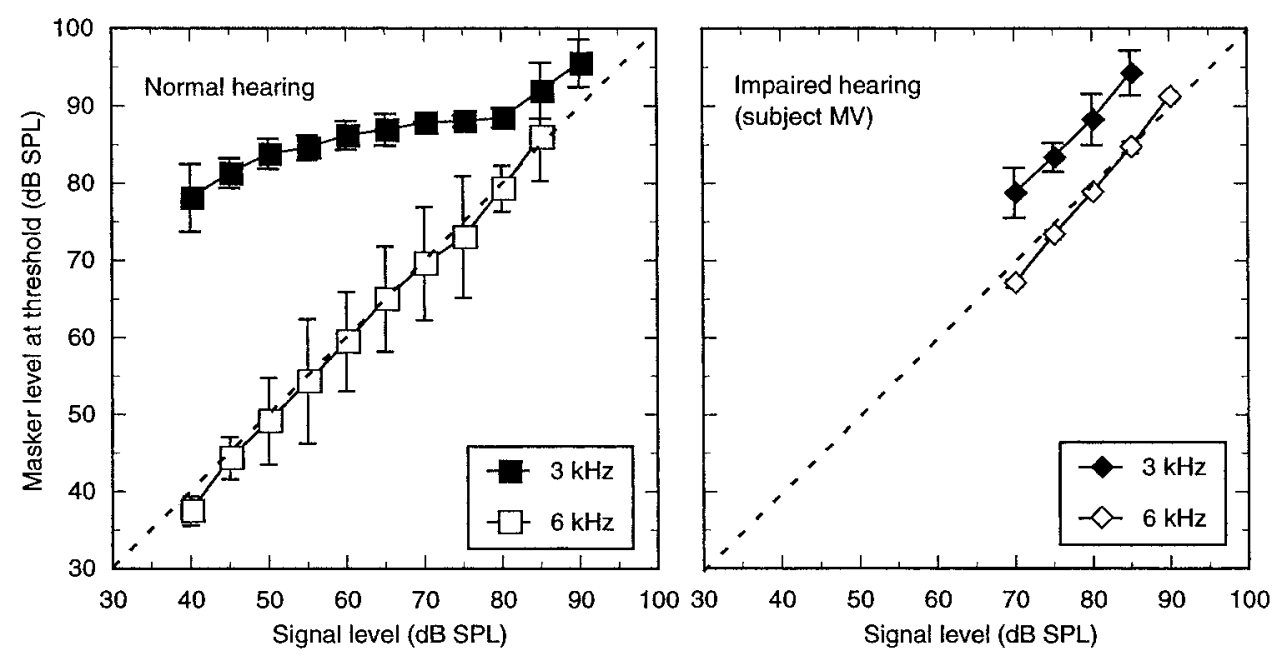

Figure 9. On- and off-frequency forward masking, used to estimate BM compression. The data in the left panel are the mean thresholds for three normal-hearing listeners. The data in the right panel are from one of the hearing-impaired listeners. The signal and on-frequency masker were at $6 \mathrm{kHz}$ and the off-frequency masker was at $3 \mathrm{kHz}$. The data are taken from Oxenham and Plack (1997).
$12 \mathrm{~dB}$ when large spectral dips were introduced in the noise. In contrast, a group of young hearingimpaired listeners derived much less benefit from the temporal and large spectral dips with only $2-\mathrm{dB}$ and 5-dB improvements, respectively. Combining both temporal and spectral dips in the noise, and substituting the noise for a single talker also produced much less improvement for hearing-impaired listeners than it did for normal-hearing listeners. Overall, speech reception thresholds in steady-state noise were somewhat poorer in the hearing-impaired than in the normal-hearing listeners, but the difference between the groups became much greater in the more complex masking backgrounds. Thus steady-state noise, so often used in laboratory studies, may underestimate the effects of cochlear hearing loss in more complex everyday acoustic environments (Bacon, Opie, \& Montoya, 1998; Festen \& Plomp, 1990).

\section{Behavioral Measures of Cochlear Nonlinearity}

The above-mentioned examples show that changes in BM nonlinearity can account for many of the difficulties faced by hearing-impaired listeners. The ability to estimate residual BM nonlinearity in individual listeners might therefore be of some diagnostic use. One of the first explicit behavioral estimates of the BM's input-output function was provided by Oxenham and Plack (1997). They took advantage of the physiological finding that the response of the BM to tones well below the CF of the measurement point is linear (Ruggero et al., 1997; Fig. 4). A comparison was made between the growth of masking with a masker at the signal frequency (on-frequency masker) and the growth of masking with a masker well below the signal frequency (off-frequency masker). The rationale was that, at the place corresponding to the signal frequency, the response to the on-frequency masker would be compressive, whereas the response to the off-frequency masker would be linear. By comparing the two slopes, it should therefore be possible to estimate the amount of compression. To avoid nonlinear interactions between the masker and signal (such as suppression) Oxenham and Plack (1997) used forward masking. This made the results more comparable to physiological studies, where generally only one tone is presented at a time. The mean results from three normal-hearing listeners are presented in the left panel of Figure 9. The growth of masking for the on-frequency masker is essentially linear, with a $10-\mathrm{dB}$ increase in signal level being matched by a $10-\mathrm{dB}$ increase in the masker level needed to mask the signal. However, the growth of masking for the off-frequency masker is very shallow; between signal levels of 50 and $80 \mathrm{~dB}$ SPL, a $30-\mathrm{dB}$ increase in signal level results in approximately a 5 -dB increase in masker level. Note that in this experiment, the level of the forward masker was varied to mask the fixed-level signal, and so masker level is plotted as a function of signal level.

The pattern of results for the normal-hearing listeners in Figure 9 can be understood in terms of the model shown in Figure 6, as follows. The signal produces maximum stimulation at the place along the BM with a CF close to the signal's frequency, so we assume that the signal is detected there. In the on-frequency case, the signal and the masker are at similar levels and so are compressed in the same way. Thus, a given increase in signal level will be matched by the same increase in masker level. In the off-frequency case, the signal (which is at CF) is compressed, whereas the masker (which is well below $\mathrm{CF}$ ) is not compressed. In terms of $\mathrm{BM}$ excitation at the place with a CF corresponding to the 
signal frequency, a 10- $\mathrm{dB}$ increase in signal level might only produce a $2-\mathrm{dB}$ increase in $\mathrm{BM}$ response. This would be matched by a $2-\mathrm{dB}$ increase in masker level because the response to the masker is linear. In other words, a 10-dB change in signal level produces only a 2-dB change in masker level. Thus, the slope of the off-frequency masking function provides an estimate of BM compression. Based on a slope of approximately 0.17 , the estimate of $\mathrm{BM}$ compression derived by Oxenham and Plack was approximately 6:1 at medium levels and became more linear at very low and very high levels. The results are in reasonably good agreement with physiological measurements of BM compression in other mammals (Ruggero et al., 1997; Russell \& Nilsen, 1997). Furthermore, recent work using distortion product otoacoustic emissions (DPOAEs) in humans suggests a similar pattern of results, with a linear response at very low input levels $(<20 \mathrm{~dB}$ SPL $)$ and a maximum compression ratio of between $3: 1$ and 4:1 at moderate levels, before becoming more linear again at high levels (Dorn, Konrad-Martin, Neely, Keefe, Cyr, \& Gorga, 2001). The slightly lower compression ratio found for DPOAEs may be related to the simultaneous presence of multiple tones, and the resultant suppression effects.

The results from one of the three listeners with cochlear hearing impairment who were also tested are shown in the right panel of Figure 9. In contrast to the results from the normal-hearing listeners, both the on- and off-frequency functions appear to be linear. This suggests that BM compression is greatly reduced or absent in this listener. One problem with these data is the restricted range of levels that it was possible to test in this and the other two hearing-impaired listeners. By being limited to high levels, much of the data was at levels where BM compression is reduced even in normal-hearing listeners. However, other data collected at a lower signal frequency $(2 \mathrm{kHz}$, instead of $6 \mathrm{kHz}$ ), where the hearing losses were not so severe, showed a similar lack of compression even at lower signal levels (Oxenham \& Plack, 1997).

Nelson, Schroder, and Wojtczak (2001) proposed an alternative behavioral measure of BM compression. They kept the signal fixed at a low level and varied the temporal gap between the forward masker and the signal. They also used a comparison between on-frequency and off-frequency masking to derive an estimate of $\mathrm{BM}$ compression. One advantage of this technique is that, because the signal is fixed at a low level, it is more likely that listeners are able to use only the region of the BM around the $\mathrm{CF}$ to detect the signal. Furthermore, because the place of peak excitation can vary with level (Rhode \& Recio, 2000), a technique that keeps the signal level constant is more in line with physiological studies that keep the place of measurement constant. The two measurement techniques (Nelson et al., 2001; Oxenham \& Plack, 1997) seem to produce results that are broadly consistent with one another.

\section{Relations between Different Measures of Hearing Loss}

The possibility of estimating BM compression in humans is encouraging; it confirms strong links between peripheral physiology and our perceptions. One long-term aim of such work is to provide a diagnostic tool that would be useful in a clinical setting. For instance, knowing how much BM compression an individual listener still had might determine the amount of compression that would be suitable to include within a hearing aid. However, the techniques outlined earlier are at present totally unsuitable for use in the clinic; it is not unusual for a single listener to spend 30 hours in the booth (although not all at once!) to collect the necessary data, allowing for practice effects and the like. Recent work suggests that the measurement of DPOAEs may provide a noninvasive window on these aspects of cochlear function that can be performed in a short time frame and without the need for behavioral responses (Gorga, Neely, Dorn, \& Konrad-Martin, 2002). Another advantage is that they can be used in patients (such as infants) who cannot make responses in behavioral experiments. However, it has yet to be shown that either behavioral or otoacoustic-emissions testing can provide a measure of BM compression that takes a sufficiently short time and is accurate enough to be used in the clinic.

Before investing in additional research to find a clinically suitable test of $\mathrm{BM}$ compression, it is necessary to ask whether some current test might already fulfill that requirement. For instance, why go to all the trouble of determining the amount of $\mathrm{BM}$ compression, if an audiogram will provide basically the same information? The answer is that the amount of hearing loss reflected in the audiogram may come from several different sources, only one of them being a loss of (or damage to) the OHCs, and the resulting loss of $\mathrm{BM}$ compression. These different sources may well have different effects on our sound perception and so it may be better to address them in different ways.

For example, consider loudness recruitment. It is known that listeners with the same audiometric thresholds can exhibit different amounts of loudness recruitment. Some researchers have proposed that there may be two main factors underlying cochlear hearing loss, one caused by $\mathrm{OHC}$ loss or dysfunction 
and the other caused by IHC loss or dysfunction (Launer, 1995; Launer, Hohmann, \& Kollmeier, 1997; Moore \& Glasberg, 1997; Moore, Glasberg, \& Vickers, 1999). It is conjectured that OHC loss results in elevated thresholds and a loss of compression, whereas IHC loss may have an effect that is similar to simple linear attenuation (of course, deafness will result from a total loss of IHCs). Although there is little physiological evidence for such a division, especially with regard to the IHCs, the distinction between linear and nonlinear processes provides a potentially useful initial framework within which research questions can be posed. In any case, Moore and colleagues (Moore \& Glasberg, 1997; Moore, Glasberg, \& Vickers, 1999) have shown that by combining the amount of assumed IHC and OHC loss in different proportions, most observed patterns of loudness recruitment could be simulated. Within this framework, the audiometric threshold reflects the summed effect of IHC and OHC loss, but provides no way of distinguishing between them.

Does the improvement in describing loudness recruitment with two components really reflect different underlying mechanisms, or is it just a consequence of having more free parameters? If the distinction made between the different underlying components were real, one would expect to find strong correlations between measures thought to reflect the same mechanism, and weak correlations between measures thought to reflect different mechanisms. This proposition was tested in a study of bilateral and unilateral hearing-impaired listeners by Moore, Vickers, Plack, and Oxenham (1999). They measured audiometric thresholds, auditory filter shapes, growth of forward masking for on- and off-frequency maskers, and loudness functions. The hypothesis was that frequency selectivity (as measured by auditory filter shape), slopes from growth of masking (Oxenham \& Plack, 1997), and loudness recruitment are all affected by the same underlying mechanism, namely the OHCs, whereas audiometric thresholds are determined by a combination of IHC loss, OHC loss, and possibly other factors. If so, then the results from the first three measures should be highly correlated with each other, but not so highly correlated with audiometric threshold. Overall, the predictions were confirmed. As in previous studies, the correlation between auditory filter bandwidth and audiometric threshold was not very high $(r=0.58)$; for the same hearing loss of approximately $60 \mathrm{~dB}$, some filter bandwidths were less than twice the normal bandwidth, whereas others were wider by a factor of nearly 10 . In contrast, the correlation between the filter bandwidths and the slopes from the growth of forward masking were highly correlated ( $r=0.92)$, providing strong sup- port for the idea that the two measures reflect the same underlying mechanism. The correlation between frequency selectivity and the $\mathrm{OHC}$ parameter in the loudness model was intermediate $(r=0.75)$. This may be because of the inherent variability associated with loudness judgments, or because the assumption of one linear and one nonlinear component to hearing loss is too simplistic. In any case, the two objective masking measures (filter shape and growth of masking), which are both thought to reflect the same changes in cochlear nonlinearity, were highly correlated.

\section{Temporary Hearing Loss: Insights from Normal-Hearing Listeners}

It is possible to gain considerable insight into the role of nonlinear cochlear processing in auditory perception by studying the effects of temporary hearing loss. This can be done, for example, by studying the effects of aspirin (acetylsalicylic acid), which, when taken in moderate doses, can cause a temporary hearing loss by affecting the OHCs (Russell \& Schauz, 1995; Shehata, Brownell, \& Dieler, 1991; Stypulkowski, 1990). One consequence of the ingestion of moderate doses of aspirin is an elevation of absolute thresholds, usually on the order of approximately 5 to $20 \mathrm{~dB}$ (for a review, see Cazals, 2000). Although the magnitude of this loss is not especially great, it nevertheless provides an interesting opportunity to examine the effects of (temporary) disruption solely to the OHCs.

The increase in absolute thresholds caused by aspirin can be understood in terms of a reduction in the gain of the cochlear amplifier provided by the OHCs. We are unaware of any measures of the effects of aspirin on loudness recruitment, although quinine, which causes a temporary loss that is also thought to reflect a disruption of the $\mathrm{OHCs}$, raises absolute threshold without changing the uncomfortable loudness level (Berninger, Karlsson, \& Alván, 1998). Thus, it appears that temporary OHC damage results in elevated absolute thresholds and loudness recruitment. This is, of course, what one would expect, given that the OHCs provide little or no gain at high stimulus levels.

Aspirin is also known to reduce or impair frequency selectivity. This has been observed in paradigms designed to measure critical bands by loudness comparisons (Bonding, 1979), psychophysical tuning curves (Beveridge \& Carlyon, 1996), and auditory filter shapes (Carlyon \& Butt, 1993; Hicks $\&$ Bacon, 1999). This impairment can be understood in terms of a reduction in the frequency-specific gain provided by the OHCs, and it mimics the effects of 
permanent loss on frequency selectivity discussed earlier.

There is also evidence that aspirin has some effect on measures of temporal processing. For example, aspirin has been shown to reduce temporal integration (Hicks \& Bacon, 1999; McCabe \& Dey, 1965; McFadden, Plattsmier, \& Pasanen, 1984). Moreover, McFadden et al. (1984) found that aspirin reduced the rate of recovery from a forward masker in two of their three listeners with the most severe (approximately 10 to $20 \mathrm{~dB}$ ) hearing loss. This is consistent with the expected effect of reduced compression on forward masking (Fig. 7). McFadden et al. (1984) also showed that aspirin increased the gap-detection threshold for noise bands at low (but not high) sound pressure levels. All of these effects of aspirin on temporal processing are broadly consistent with the effects of permanent sensorineural hearing loss, and can at least partly be understood in terms of a reduction in cochlear compression.

As discussed earlier, it is possible to obtain an estimate of cochlear compression via a forwardmasking paradigm (Nelson et al., 2001; Oxenham \& Plack, 1997; Fig. 9). Hicks and Bacon (Reference Note 1) have shown that aspirin reduces the estimated amount of compression in a way that is qualitatively similar to the way in which quinine reduces the amount of compression measured directly at the BM (Ruggero et al., 1996). These results thus provide strong support for a close connection between the amount of compression estimated psychophysically and that measured at the BM, and they underscore the importance of normal $\mathrm{OHC}$ function for compression.

The results presented in this section strengthen the argument for a connection between normalfunctioning $\mathrm{OHCs}$, leading to a nonlinear cochlear response, and certain measures of auditory perception.

\section{What BM Compression Cannot Account For}

This paper has reviewed a number of apparently disparate phenomena that can be accounted for by the changes in BM compression that occur with $\mathrm{OHC}$ loss or dysfunction. Although this provides a powerful framework for understanding many of the difficulties experienced by hearing-impaired listeners, it must be remembered that there are other, possibly important aspects of hearing loss. One has been termed IHC loss, and has been used to account for aspects of loudness recruitment (Moore \& Glasberg, 1997; Moore, Glasberg, \& Vickers, 1999) and the behavioral consequences of so-called dead regions in the cochlea (Moore \& Alcantara, 2001; Moore, Huss, Vickers, Glasberg, \& Alcantara, 2000).
There are clearly also many forms of retrocochlear hearing loss. Auditory neuropathy (Kraus et al., 2000; Sininger \& Starr, 2001; Starr, Picton, Sininger, Hood, \& Berlin, 1996) is a topic of considerable current interest. Such patients present with normal cochlear function but abnormal or absent auditory brain stem responses. An interesting finding in auditory neuropathy patients has been extremely poor temporal resolution, as measured using gap detection, modulation detection, and frequency discrimination (Zeng, Oba, Garde, Sininger, \& Starr, 1999, 2001). Clearly these deficits are different from the temporal-processing deficits expected from a loss of BM nonlinearity. Future research should help us to devise behavioral and other methods to distinguish between the different sources underlying a given hearing loss in a much more sophisticated way than is possible today.

\section{Summary ANd OUTLOOK}

This paper has reviewed a range of psychoacoustic results that can be explained in terms of known characteristics of cochlear physiology, in particular the nonlinear input-output function of the BM. These results were elaborated in terms of their potential effect on listening in the everyday environment. Overall, many common perceptual aspects of cochlear hearing impairment can be understood in terms of changes in the BM input-output function. These include raised audiometric thresholds, loudness recruitment, poorer frequency selectivity, and effectively poorer temporal processing under certain conditions.

A major goal of psychoacoustic research into hearing impairment is to explain the perceptual difficulties of hearing-impaired patients in terms of underlying physiological mechanisms. The hope is that by understanding the underlying mechanisms we will better be able to compensate for the deficits. Of primary importance is the ability of listeners to understand speech and hence to be able to communicate freely. There have been a number of attempts to correlate speech reception abilities with more basic psychoacoustic tasks (Dreschler \& Plomp, 1980, 1985; Festen \& Plomp, 1981, 1983; Noordhoek, Houtgast, \& Festen, 2001; Tyler, Summerfield, Wood, \& Fernandes, 1982). In these studies, although certain psychoacoustic tasks, such as gap detection, have been associated with broad categories, such as temporal processing, there has been little attempt to pinpoint any underlying mechanisms. In this way, some of the resulting conclusions may be less informative than they could be or, in some cases, may even be misleading. For instance, in a recent such study (Noordhoek, Houtgast, Fes- 
ten, 2001), forward masking was discussed as a measure of temporal resolution, distinct from compression. However, as shown in this paper, compression can strongly affect forward masking in a way that has nothing to do with temporal processing per se. Similar arguments can be applied to frequency selectivity and peripheral compression, as one important aspect of cochlear nonlinearity is the sharp tuning it provides at low levels. The compressive nonlinearity could therefore be viewed as a byproduct of the level-dependent frequency selectivity, or vice versa. In any case the two effects, sharp frequency tuning and the compressive input-output function, are not separable in terms of cochlear mechanics and are probably best regarded as two sides of the same coin. Thus, caution is required in labeling certain psychoacoustic tasks as being related to either frequency selectivity or peripheral compression (Noordhoek et al., 2001); single tasks do not necessarily reflect single processes.

By using accurate cochlear models and providing quantitative simulations, we can begin to predict how certain underlying mechanisms might affect perceptual measures. The effects of BM compression are probably the easiest to treat in this way. However, the hope is that more accurate models of cochlear processing will lead to a deeper understanding of how the various physiological changes that cause cochlear hearing loss can help to account for the changes in perception associated with hearing impairment. The ability to break down a single hearing loss into its constituent components may help us account for at least a part of the large variability in performance between different hearing-impaired listeners and may enable us to tailor the treatment of individual losses more successfully to the individual patient.

\section{ACKNowledgments:}

The study was supported by the National Institute on Deafness and other Communication Disorders of the National Institutes of Health (Grants R01 DC 03909 and R01 DC 01376). The authors thank the collaborators in much of the research described, particularly Brian Moore and Chris Plack, and also Li Liu for her help with some of the figures.

Address for correspondence: Andrew J. Oxenham, Ph.D., Research Laboratory of Electronics, Bldg. 36-763, Massachusetts Institute of Technology, Cambridge, MA 02139-4307. E-mail: oxenham@mit.edu.

Received February 24, 2003; accepted May 13, 2003

\section{REFERENCES}

Bacon, S. P., \& Gleitman, R. (1992). Modulation detection in subjects with relatively flat hearing losses. Journal of Speech and Hearing Research, 35, 642-653.
Bacon, S. P., Opie, J. M., \& Montoya, D. Y. (1998). The effects of hearing loss and noise masking on the masking release for speech in temporally complex backgrounds. Journal of Speech, Language, and Hearing Research, 41, 549-563.

Bacon, S. P., \& Oxenham, A. J. (in press). Psychophysical manifestations of cochlear compression: Hearing-impaired listeners. In S. P. Bacon, R. Fay \& A. N. Popper (Eds.), Compression: From Cochlea to Cochlear Implant. New York: Springer Verlag.

Bacon, S. P., \& Viemeister, N. F. (1985). Temporal modulation transfer functions in normal-hearing and hearing-impaired subjects. Audiology, 24, 117-134.

Baer, T., \& Moore, B. C. J. (1993). Effects of spectral smearing on the intelligibility of sentences in the presence of noise. Journal of the Acoustical Society of America, 94, 1229-1241.

Berninger, E., Karlsson, K. K., \& Alván, G. (1998). Quinine reduces the dynamic range of the human auditory system. Acta Otolaryngologia, 118, 46-51.

Beveridge, H. A., \& Carlyon, R. P. (1996). Effects of aspirin on human psychophysical tuning curves in forward and simultaneous masking. Hearing Research, 99, 110-118.

Bonding, P. (1979). Critical bandwidth in patients with a hearing loss induced by salicylates. Audiology, 18, 133-144.

Buus, S., \& Florentine, M. (2001). Growth of loudness in listeners with cochlear hearing losses: Recruitment reconsidered. Journal of the Association for Research in Otolaryngology, 3, 120139.

Carlyon, R. P., \& Butt, M. (1993). Effects of aspirin on human auditory filters. Hearing Research, 66, 233-244.

Cazals, Y. (2000). Auditory sensori-neural alterations induced by salicylate. Progress in Neurobiology, 62, 583-631.

Cherry, E. C. (1953). Some experiments on the recognition of speech, with one and two ears. Journal of the Acoustical Society of America, 25, 975-979.

Dau, T., Püschel, D., \& Kohlrausch, A. (1996). A quantitative model of the 'effective' signal processing in the auditory system. I. Model structure. Journal of the Acoustical Society of America, 99, 3615-3622.

de Boer, E. (1985). Auditory time constants: A paradox? In A. Michelsen (Ed.), Time Resolution in Auditory Systems (pp. 141-158). Berlin: Springer-Verlag.

Derleth, R. P., Dau, T., \& Kollmeier, B. (2001). Modeling temporal and compressive properties of the normal and impaired auditory system. Hearing Research, 159, 132-149.

Dorn, P. A., Konrad-Martin, D., Neely, S. T., Keefe, D. H., Cyr, E., \& Gorga, M. P. (2001). Distortion product otoacoustic emission input/output functions in normal-hearing and hearing-impaired human ears. Journal of the Acoustical Society of America, 110, 3119-3131.

Dreschler, W. A., \& Plomp, R. (1980). Relations between psychophysical data and speech perception for hearing-impaired subjects. I. Journal of the Acoustical Society of America, 68, 1608-1615.

Dreschler, W. A., \& Plomp, R. (1985). Relations between psychophysical data and speech perception for hearing-impaired subjects. II. Journal of the Acoustical Society of America, 78, 1261-1270.

Dubno, J. R., \& Schaefer, A. B. (1995). Frequency selectivity and consonant recognition for hearing-impaired and normal-hearing listeners with equivalent masked thresholds. Journal of the Acoustical Society of America, 97, 1165-1174.

Eggermont, J. J. (2002). Temporal modulation transfer functions in cat primary auditory cortex: Separating stimulus effects from neural mechanisms. Journal of Neurophysiology, 87, 305-321.

Evans, E. F. (1974). Auditory frequency selectivity and the cochlear nerve. In E. Zwicker \& E. Terhardt (Eds.), Facts and Models in Hearing (pp. 118-129). New York: Springer-Verlag. 
Festen, J. M., \& Plomp, R. (1981). Relations between auditory functions in normal hearing. Journal of the Acoustical Society of America, 70, 356-369.

Festen, J. M., \& Plomp, R. (1983). Relations between auditory functions in impaired hearing. Journal of the Acoustical Society of America, 73, 652-662.

Festen, J. M., \& Plomp, R. (1990). Effects of fluctuating noise and interfering speech on the speech reception threshold for impaired and normal hearing. Journal of the Acoustical Society of America, 88, 1725-1736.

Fitzgibbons, P. J., \& Gordon-Salant, S. (1987). Temporal gap resolution in listeners with high-frequency sensorineural hearing loss. Journal of the Acoustical Society of America, 81, 133-137.

Fitzgibbons, P. J., \& Wightman, F. L. (1982). Gap detection in normal and hearing-impaired listeners. Journal of the Acoustical Society of America, 72, 761-765.

Florentine, M., Buus, S., Scharf, B., \& Zwicker, E. (1980). Frequency selectivity in normally-hearing and hearing-impaired observers. Journal of Speech and Hearing Research, 23, 643669.

Glasberg, B. R., \& Moore, B. C. J. (1992). Effects of envelope fluctuations on gap detection. Hearing Research, 64, 81-92.

Glasberg, B. R., Moore, B. C. J., \& Bacon, S. P. (1987). Gap detection and masking in hearing-impaired and normal-hearing subjects. Journal of the Acoustical Society of America, 81, 1546-1556.

Gorga, M. P., Neely, S. T., Dorn, P. A., \& Konrad-Martin, D. (2002). The use of distortion product otoacoustic emission suppression as an estimate of response growth. Journal of the Acoustical Society of America, 111, 271-284.

Harrison, R. V. (1981). Rate-versus-intensity functions and related AP responses in normal and pathological guinea pig and human cochleas. Journal of the Acoustical Society of America, 70, 1036-1044.

Hicks, M. L., \& Bacon, S. P. (1999). Effects of aspirin on psychophysical measures of frequency selectivity, two-tone suppression, and growth of masking. Journal of the Acoustical Society of America, 106, 1436-1451.

Holube, I., \& Kollmeier, B. (1996). Speech intelligibility prediction in hearing-impaired listeners based on a psychoacoustically motivated perception model. Journal of the Acoustical Society of America, 100, 1703-1717.

Irwin, R. J., Hinchcliff, L. K., \& Kemp, S. (1981). Temporal acuity in normal and hearing-impaired listeners. Audiology, 20, 234243.

Kraus, N., Bradlow, A. R., Cheatham, M. A., Cunningham, J., King, C. D., Koch, D. B., Nicol, T. G., McGee, T. G., Stein, L. K., \& Wright, B. A. (2000). Consequences of neural asynchrony: A case of auditory neuropathy. Journal of the Association for Research in Otolaryngology, 1, 33-45.

Launer, S. (1995). Loudness Perception in Listeners with Sensorineural Hearing Impairment. Unpublished Ph.D. thesis, Oldenburg University, Oldenburg, Germany.

Launer, S., Hohmann, V., \& Kollmeier, B. (1997). Modeling loudness growth and loudness summation in hearing-impaired listeners. In W. Jesteadt (Ed.), Modeling Sensorineural Hearing Loss (pp. 175-186). Mahwah, NJ: Erlbaum.

Leek, M. R., \& Summers, V. (1993). Auditory filter shapes of normal-hearing and hearing-impaired listeners in continuous broadband noise. Journal of the Acoustical Society of America, 94, 3127-3137.

Levitt, H. (in press). Compression amplification. In S. P. Bacon, R. Fay \& A. N. Popper (Eds.), Compression: From Cochlea to Cochlear Implant. New York: Springer Verlag.
McCabe, P. A., \& Dey, F. L. (1965). The effect of aspirin upon auditory sensitivity. The Annals of Otology Rhinology \& Laryngology, 74, 312-325.

McFadden, D., Plattsmier, H. S., \& Pasanen, E. G. (1984). Temporary hearing loss induced by combinations of intense sounds and nonsteroidal anti-inflammatory drugs. American Journal of Otolaryngology, 5, 235-241.

Moore, B. C. J. (1985). Frequency selectivity and temporal resolution in normal and hearing impaired listeners. British Journal of Audiology, 19, 189-201.

Moore, B. C. J. (1998). Cochlear Hearing Loss. London: Whurr.

Moore, B. C. J., \& Alcantara, J. I. (2001). The use of psychophysical tuning curves to explore dead regions in the cochlea. Ear and Hearing, 22, 268-278.

Moore, B. C. J., \& Glasberg, B. R. (1988). Gap detection with sinusoids and noise in normal, impaired and electrically stimulated ears. Journal of the Acoustical Society of America, 83, 1093-1101.

Moore, B. C. J., \& Glasberg, B. R. (1997). A model of loudness perception applied to cochlear hearing loss. Auditory Neuroscience, 3, 289-311.

Moore, B. C. J., Glasberg, B. R., \& Vickers, D. A. (1999). Further evaluation of a model of loudness perception applied to cochlear hearing loss. Journal of the Acoustical Society of America, 106, 898-907.

Moore, B. C. J., Huss, M., Vickers, D. A., Glasberg, B. R., \& Alcantara, J. I. (2000). A test for the diagnosis of dead regions in the cochlea. British Journal of Audiology, 34, 205-224.

Moore, B. C. J., \& Oxenham, A. J. (1998). Psychoacoustic consequences of compression in the peripheral auditory system. Psychological Review, 105, 108-124.

Moore, B. C. J., Vickers, D. A., Plack, C. J., \& Oxenham, A. J. (1999). Inter-relationship between different psychoacoustic measures assumed to be related to the cochlear active mechanism. Journal of the Acoustical Society of America, 106, 27612778.

Narayan, S. S., Temchin, A. N., Recio, A., \& Ruggero, M. A. (1998). Frequency tuning of basilar membrane and auditory nerve fibers in the same cochleae. Science, 282, 1882-1884.

Nelson, D. A., \& Freyman, R. L. (1987). Temporal resolution in sensorineural hearing-impaired listeners. Journal of the Acoustical Society of America, 81, 709-720.

Nelson, D. A., Schroder, A. C., \& Wojtczak, M. (2001). A new procedure for measuring peripheral compression in normalhearing and hearing-impaired listeners. Journal of the Acoustical Society of America, 110, 2045-2064.

Noordhoek, I. M., Houtgast, T., \& Festen, J. M. (2001). Relations between intelligibility of narrow-band speech and auditory functions, both in the $1-\mathrm{kHz}$ frequency region. Journal of the Acoustical Society of America, 109, 1197-1212.

Oxenham, A. J., \& Bacon, S. P. (in press). Psychophysical manifestations of cochlear compression: Normal-hearing listeners. In S. P. Bacon, R. Fay \& A. N. Popper (Eds.), Compression: From Cochlea to Cochlear Implant. New York: Springer Verlag.

Oxenham, A. J., \& Moore, B. C. J. (1997). Modeling the effects of peripheral nonlinearity in normal and impaired hearing. In W. Jesteadt (Ed.), Modeling Sensorineural Hearing Loss (pp. 273288). Mahwah, NJ: Erlbaum.

Oxenham, A. J., Moore, B. C. J, \& Vickers, D. A. (1997). Shortterm temporal integration: Evidence for the influence of peripheral compression. Journal of the Acoustical Society of America, 101, 3676-3687.

Oxenham, A. J., \& Plack, C. J. (1997). A behavioral measure of basilar-membrane nonlinearity in listeners with normal and impaired hearing. Journal of the Acoustical Society of America, 101, 3666-3675. 
Peters, R. W., Moore, B. C. J, \& Baer, T. (1998). Speech reception thresholds in noise with and without spectral and temporal dips for hearing-impaired and normally hearing people. Journal of the Acoustical Society of America, 103, 577-587.

Qin, M. K., \& Oxenham, A. J. (2003). Effects of simulated cochlear-implant processing on speech reception in fluctuating maskers. Journal of the Acoustical Society of America, 114, $446-454$.

Rhode, W. S. (1971). Observations of the vibration of the basilar membrane in squirrel monkeys using the Mössbauer technique. Journal of the Acoustical Society of America, 49, 1218 1231.

Rhode, W. S., \& Recio, A. (2000). Study of mechanical motions in the basal region of the chinchilla cochlea. Journal of the Acoustical Society of America, 107, 3317-3332.

Robles, L., \& Ruggero, M. A. (2001). Mechanics of the mammalian cochlea. Physiological Reviews, 81, 1305-1352.

Ruggero, M. A., \& Rich, N. C. (1991). Furosemide alters organ of Corti mechanics: Evidence for feedback of outer hair cells upon the basilar membrane. Journal of Neuroscience, 11, 10571067.

Ruggero, M. A., Rich, N. C., \& Recio, A. (1996). The effect of intense acoustic stimulation on basilar-membrane vibrations. Auditory Neuroscience, 2, 329-345.

Ruggero, M. A., Rich, N. C., Recio, A., Narayan, S. S., \& Robles, L. (1997). Basilar-membrane responses to tones at the base of the chinchilla cochlea. Journal of the Acoustical Society of America, 101, 2151-2163.

Ruggero, M. A., Rich, N. C., Robles, L., \& Recio, A. (1996). The effects of acoustic trauma, other cochlear injury, and death on basilar-membrane responses to sound. In A. Axelsson, H. Borchgrevink, R. P. Hamernik, P. Hellström, D. Henderson \& R. J. Salvi (Eds.), Scientific Basis of Noise-Induced Hearing Loss (pp. 23-35). New York: Thieme.

Ruggero, M. A., Robles, L., Rich, N. C., and Recio, A. (1992). Basilar membrane responses to two-tone and broadband stimuli. Philosophical Transactions of the Royal Society, London B, 336, 307-315.

Russell, I. J., \& Nilsen, K. E. (1997). The location of the cochlear amplifier: Spatial representation of a single tone on the guinea pig basilar membrane. Proceedings of the National Academy of Sciences, 94, 2660-2664.

Russell, I. J., \& Schauz, C. (1995). Salicylate ototoxicity: Effects on stiffness and electromotility of outer hair cells isolated from the guinea pig cochlea. Auditory Neuroscience, 1, 309-319.

Sellick, P. M., Patuzzi, R., \& Johnstone, B. M. (1982). Measurement of basilar membrane motion in the guinea pig using the Mössbauer technique. Journal of the Acoustical Society of America, 72, 131-141.

Shehata, W. E., Brownell, W. E., \& Dieler, R. (1991). Effects of salicylate on shape, electromotility and membrane character- istics of isolated outer hair cells from guinea pig cochlea. Acta Otolaryngologica, 111, 707-718.

Sininger, Y., \& Starr, A. (Eds.). (2001). Auditory Neuropathy. San Diego: Singular.

Snell, K. B., \& Frisina, D. R. (2000). Relationships among age-related differences in gap detection and word recognition. Journal of the Acoustical Society of America, 107, 1615-1626.

Starr, A., Picton, T. W., Sininger, Y., Hood, L. J., \& Berlin, C. I. (1996). Auditory neuropathy. Brain, 119, 741-753.

Stypulkowski, P. H. (1990). Mechanisms of salicylate ototoxicity. Hearing Research, 46, 113-146.

ter Keurs, M., Festen, J. M., \& Plomp, R. (1992). Effect of spectral envelope smearing on speech reception. Journal of the Acoustical Society of America, 91, 2872-2880.

ter Keurs, M., Festen, J. M., \& Plomp, R. (1993). Effect of spectral envelope smearing on speech reception. II. Journal of the Acoustical Society of America, 93, 1547-1552.

Tyler, R. S., Hall, J. W., Glasberg, B. R., Moore, B. C. J., \& Patterson, R. D. (1984). Auditory filter asymmetry in the hearing impaired. Journal of the Acoustical Society of America, 76, 1363-1368.

Tyler, R. S., Summerfield, A. Q., Wood, E. J., \& Fernandes, M. A. (1982). Psychoacoustic and phonetic temporal processing in normal and hearing-impaired listeners. Journal of the Acoustical Society of America, 72, 740-752.

Viemeister, N. F., \& Wakefield, G. H. (1991). Temporal integration and multiple looks. Journal of the Acoustical Society of America, 90, 858-865.

Yates, G. K. (1990). Basilar membrane nonlinearity and its influence on auditory nerve rate-intensity functions. Hearing Research, 50, 145-162.

Yates, G. K., Winter, I. M., \& Robertson, D. (1990). Basilar membrane nonlinearity determines auditory nerve rate-intensity functions and cochlear dynamic range. Hearing Research, $45,203-219$.

Zeng, F. G., Oba, S., Garde, S., Sininger, Y., \& Starr, A. (1999). Temporal and speech processing deficits in auditory neuropathy. Neuroreport, 10, 3429-3435.

Zeng, F. G., Oba, S., Garde, S., Sininger, Y., \& Starr, A. (2001). Psychoacoustics and speech perception in auditory neuropathy. In Y. Sininger \& A. Starr (Eds.), Auditory Neuropathy (pp. 141-164). San Diego: Singular.

\section{Reference Note}

1 Hicks, M. L., \& Bacon, S. P. (2000). The effects of aspirin on a psychophysical estimate of basilar membrane compression. Proceedings of the 139th meeting of the Acoustical Society of America, Atlanta, GA. 